\section{Nociones e ideas en torno al universalismo en salud: derivas en la trama intergubernamental del Gran Buenos Aires (Argentina) entre 2003 y 2011}

\author{
Concepts and ideas concerning universal \\ healthcare: results of the intergovernmental \\ arrangement in Greater Metropolitan Buenos \\ Aires, Argentina, from 2003 to 2011
}

\author{
Noções e ideias em torno ao universalismo em \\ saúde: derivas na trama intergovernamental \\ da Grande Buenos Aires, Argentina, entre \\ 2003 e 2011
}

\section{Resumen}

En el contexto del marco institucional federal y fuertemente fragmentado de Argentina, este artículo analiza las nociones e ideas que los actores gubernamentales organizan alrededor de los instrumentos de politica sanitaria en los tres niveles de gobierno diferenciados. A partir de este enfoque, se pretende indagar acerca de las convergencias, divergencias y tensiones que atraviesan el ejercicio del derecho a la salud. El análisis se organiza en tres dimensiones del universalismo que se convirtieron en desafíos de la politica nacional durante el periodo analizado: facilitación en el acceso a los servicios, aseguramiento de la cobertura y garantía de un conjunto de beneficios explícitos para toda la población. En torno a estos desafíos, los actores deconstruyen y reconstruyen el significado de las políticas orientadas a la universalidad en salud, según los problemas que forman parte de su agenda, las ideas previas a la llegada de los programas (y a partir de las cuales conciben los cambios) y la lógica política a través de la que viabilizan sus decisiones. Esta perspectiva busca transcender las nociones técnicas que justifican los programas, con el fin de captar la dimensión política de la implementación, entendida como una construcción social compleja, que enfrenta también problemas estructurales que componen la agenda en cada nivel de gobierno, en relación a la provisión de servicios de salud.

Politica de Salud; Acceso Universal a los Servicios de Salud; Federalismo
Magdalena Chiara 1

doi: 10.1590/0102-311X00002018

\author{
Correspondencia \\ M. Chiara \\ Universidad Nacional de General Sarmiento. \\ Lavalle 2830, San Fernando, Buenos Aires - 1646, Argentina. \\ magda.chiara@gmail.com \\ 1 Universidad Nacional de General Sarmiento, Buenos Aires, \\ Argentina.
}




\section{Introducción}

Al menos cuatro atributos convierten al sector salud argentino en un campo de singular complejidad: sus instituciones están organizadas en tres subsectores (seguridad social, privado y estatal); en este último convergen en la provisión de servicios los tres niveles gubernamentales; y desde la reforma constitucional del año 1994, todos los habitantes gozan del derecho a la provisión pública de salud. Recursos de distinta fuente concurren en el financiamiento, una multiplicidad de instituciones son responsables de la provisión de servicios, y la función de rectoría encuentra serias dificultades para articular un sistema que esté a la altura del mandato constitucional que garantiza el derecho a la salud.

En el contexto del federalismo, la distribución de las competencias en relación a salud es el cuarto atributo que distingue, en este caso, al subsector estatal. No habiendo sido delegadas por los estados federados al nivel central, las responsabilidades en materia sanitaria permanecieron en la órbita provincial y -en algunos casos- fueron transferidas (mediando un marco regulatorio o sin él) a los municipios. Como resultado de estos procesos, coexisten constituciones provinciales que definen diferentes contenidos del derecho a la salud en cada jurisdicción, donde la amplitud de la oferta de servicios y su calidad están asociadas a la capacidad de financiamiento que tiene cada jurisdicción. Esta situación se ve negativamente retroalimentada en aquellas jurisdicciones más pobres, y que tienen a su vez poblaciones que padecen mayores necesidades, dependiendo de los servicios que pueden proveer las instituciones estatales, sean éstas provinciales o municipales. Como resultado de este fenómeno de fragmentación institucional, las condiciones bajo las cuales se garantiza la provisión de servicios de salud son muy desiguales y en aquellas localidades donde se concentra la población que presenta mayores problemas sociales y sanitarios, la situación es particularmente crítica.

En un contexto institucional sumamente fragmentado y en territorios tan desiguales, las políticas orientadas hacia la universalización o, más modestamente, a cerrar las brechas en las condiciones bajo las cuales se garantiza el derecho a la salud, enfrentan ineludiblemente desafíos relativos a cómo se procesa la dinámica de las relaciones intergubernamentales.

En ese contexto, este trabajo indaga las convergencias, divergencias y tensiones que atraviesan los intentos de cambio orientados a la restauración de las condiciones más igualitarias en el ejercicio del derecho a la salud en el Gran Buenos Aires, territorio caracterizado por la doble condición de fragmentación institucional y desigualdad, entre los años 2003 y 2011.

Se trata de un periodo en el que se sucedieron distintos intentos de cambio orientados a igualar las condiciones en el ejercicio del derecho a la salud. Entre otros dispositivos nacionales se destacan el Programa Remediar y el Plan Nacer (posteriormente denominado Sumar). Reconstruyendo los contrapuntos en torno a estos dispositivos, nos interesa identificar las diferencias en los modos como los actores conciben los intentos de cambio hacia la universalidad. Se trata de un estudio cualitativo que explora la arena de la implementación desde un enfoque macro institucional, a fin de comprender el significado que le confieren los actores a las políticas en contextos institucionales determinados.

El artículo está organizado en dos apartados y una conclusión. A esta introducción le sigue un apartado teórico metodológico; una segunda parte presenta los resultados, caracterizando el contexto de fragmentación y los dispositivos de política nacional, y reconstruyendo los contrapuntos entre los actores en torno a las ideas que organizan estas reformas. Finaliza con las conclusiones.

\section{Aproximación teórico-metodológica}

La pregunta que orienta este trabajo pone en diálogo distintas contribuciones del campo de las políticas públicas, con el propósito de analizar el derrotero de las reformas en salud en la trama intergubernamental. La aproximación teórica que adoptamos busca responder a tres problemas o cuestiones que desafían a nuestra pregunta en el contexto singular del sector en Argentina.

La primera refiere a la necesidad de dar cuenta de los modos como los actores modelan sus decisiones en el proceso de las políticas. Distintos autores del neoinstitucionalismo ayudan a comprender los modos a través de los cuales los actores organizan sus preferencias en el contexto de las instituciones del bienestar; en algunos casos, estas contribuciones han puesto el foco de modo particular en los contextos federales 1,2. Esta corriente busca explicar cómo las instituciones condicionan (limitando 
y/o posibilitando) el margen de acción de los actores en el proceso de la política pública, a través de las rutinas y/o de las reglas de juego en cuyo contexto se organiza la acción pública. Más particularmente el neoinstitucionalismo histórico aportó el concepto de path dependence 3 para explicar algunas decisiones como resultado de procesos históricos, cuya trayectoria condiciona el presente y puede reforzar la recurrencia de un determinado patrón de relaciones en el futuro. Si bien esta perspectiva permite comprender las diferencias en el comportamiento de los actores en los distintos niveles (nación, provincia y municipios) 2,3,4, pone en evidencia algunas limitaciones para captar los procesos, a través de los cuales se gestan los cambios y discurren por los espacios de decisión.

La preocupación que anima nuestra pregunta se refiere al modo como se construyen los cambios orientados hacia la universalidad en salud, lo cual remite -en segundo lugar- a la cuestión acerca del papel que asumen las ideas en las políticas. Aunque la producción es importante, los estudios han concentrado su atención en los procesos de cambio que tienen lugar en el momento de formulación de la política pública, mostrando importantes lagunas, en relación a lo que sucede en la implementación 5.

La literatura presenta distintas "tipologías" para analizar las ideas que intervienen en los procesos de decisión. La propuesta de Sabatier \& Weible 6 define tres niveles en los que se despliegan las "ideas" que tienen por función "cementar" la conformación de lo que denominan "coaliciones promotoras": las ideas fundamentales (deep core beliefs), las creencias sobre las políticas públicas (policy core beliefs) y las creencias instrumentales (secondary beliefs).

Desde un enfoque cognitivo, Muller ${ }^{7}$ propone mirar a las políticas públicas como un lugar a través del cual la sociedad construye su relación con el mundo y en cuyo devenir se elaboran las representaciones que permiten entender y actuar sobre sus problemas. Esta perspectiva destaca la importancia que tienen las ideas, a la vez que restituye el carácter histórico, además de los procesos a través de los cuales se producen los "referenciales", esto es, el conjunto de ideas que organiza una política pública.

De esta manera, el análisis desplaza su atención desde la "coalición promotora" hacia la red de actores intergubernamentales, en cuyo contexto se construye ese marco de referencia, atendiendo al papel que asumen los "mediadores" en la decodificación entre el "referencial global" y el "referencial sectorial" 7. Esta lectura permite hacer más complejo el enfoque clásico acerca del papel de los "mediadores" en los análisis de las relaciones intergubernamentales, para dar cuenta de los sentidos que se construyen en el diálogo que tiene lugar en la implementación.

Retomando a Campbell, Beland 5 distingue cuatro tipos de ideas: aquellas que representan los supuestos sobre la existencia de problemas políticos y las soluciones capaces de resolverlos (policy paradigms); los supuestos y percepciones compartidas por la sociedad expresadas en los estudios de opinión pública (public sentiments); las "hojas de ruta" en las que se grafica un determinado curso de acción (programmes); y los símbolos y conceptos que usan los actores políticos para legitimar propuestas políticas y presentarlas al público y a un electorado específico (frames).

Desde esta perspectiva, las ideas que subyacen a las reformas puedan ser entendidas como "hojas de ruta" que orientan a los actores. Sin embargo, cuando las reformas son incrementales e involucran actores gubernamentales con elevados niveles de autonomía relativa, estas "hojas de ruta" se re-construyen en dinámicas que no están exentas de tensiones, disputas o conflictos, y a su vez dialogan con otras ideas en el proceso a través del cual se modela la agenda en cada nivel de gobierno. Esta lectura abre la tercera cuestión que desafía nuestra pregunta, referida al proceso de construcción de la agenda en salud en contextos de alta fragmentación.

En relación a esta cuestión, Kingdon 8 plantea que el proceso de conformación de la agenda involucra en simultáneo a tres procesos: en primer lugar, aquellos derivados de la "marcha inexorable de los problemas"; en segundo lugar, el "proceso gradual de acumulación de conocimiento y perspectivas" que fundan las "alternativas" disponibles en el mundo de los expertos; y, en tercer lugar, los procesos políticos que afectan al proceso de conformación de la agenda y que anidan en distinto tipo de fenómenos, no siempre cercanos a las comunidades de expertos.

Dada la encrucijada donde se coloca nuestra pregunta, resulta necesario adoptar un enfoque híbrido, que aborde la relación entre las ideas y las políticas públicas, incorporando al análisis el estudio acerca del poder que tienen las instituciones para representarlas e incorporarlas en los procesos concretos y las dinámicas que se producen en la conformación de la agenda en cada nivel de gobierno.

Se trata de un estudio cualitativo, basado particularmente en el análisis de entrevistas en profundidad realizadas a funcionarios nacionales, provinciales y municipales y en fuentes secundarias 
(documentos de los programas seleccionados; decretos nacionales; convenios para la implementación de los programas; e informes de gestión de los programas de salud analizados). La perspectiva del nivel municipal fue recuperada a través de 14 entrevistas semiestructuradas a informantes clave pertenecientes a municipios (Cuadro 1) que, siguiendo un criterio de diversificación, representan distintas "situaciones locales" en relación a: medidas de desempeño del sistema, porcentaje de población sin cobertura, patrón de dependencia del sistema local y transferencias supralocales.

\section{Resultados y discusión}

Es posible reconocer dos perspectivas en los estudios acerca de los avances a la universalidad en la región: aquella que problematiza la segregación de la que son objeto amplios sectores de la población 9 ; y otra, que analiza las propuestas de reforma, sus instrumentos y resultados 10 . Entre estas ellas, se abre paso un conjunto de investigaciones que examinan las reformas en salud, prestando atención a cuestiones relativas a la dinámica de los actores 11 y a la arquitectura institucional que se construye en el camino hacia la universalidad en territorios complejos y desiguales 12. Estos estudios destacan la importancia de las instituciones y la dinámica de los actores (domésticos e internacionales), al tiempo que advierten acerca de los desafíos que encierra el intento por recoger en la construcción de la política las particularidades locales y regionales.

En línea con estas preocupaciones, este trabajo pone en diálogo la perspectiva de los actores de los diferentes niveles de gobierno, respecto a las nociones e ideas en torno al universalismo en salud, y parte de un análisis situado en las condiciones institucionales históricamente construidas que demarcan los márgenes de acción en cada nivel.

\section{La política nacional en contextos de fragmentación}

Tres procesos se conjugan en la "historia larga" del subsector estatal en Argentina, dando lugar a una singular autonomía relativa de los actores gubernamentales en el nivel subnacional. Por una parte, el federalismo en salud de carácter "residual" y en cuyo contexto se fue cristalizando el lugar de cada estado provincial frente a la nación, al tiempo que cada uno de ellos fue organizando la distribución de las funciones relativas a la provisión de servicios de salud en los establecimientos estatales con sus municipios 13,14. Un segundo proceso refiere a la ampliación exponencial y centralizada de la infraestructura hospitalaria desde la jurisdicción nacional a mediados de siglo pasado, fuerza contraria al federalismo que abonó, aunque de manera efímera, la construcción de márgenes de autonomía relativa del gobierno nacional respecto a las provincias. Esta "historia larga" culmina muy tempranamente con un tercer proceso que revierte al anterior, la transferencia de los servicios de salud hacia las provincias, reforma motivada más por las necesidades de resolver el conflicto financiero entre la nación y los estados provinciales que por la preocupación por acercar los servicios a las preferencias de la población. En el contexto del federalismo, este proceso hizo emerger a los gobernadores (y a sus ministros) como responsables (aunque debilitados por los resultados fiscales de aquella transferencia) de la provisión de servicios de salud en los establecimientos.

En el contexto de estos tres procesos, puede dilucidarse el significado que encierra la aparente contradicción entre gobiernos provinciales, limitados en su capacidad de acción (situación derivada de la fragilidad financiera que resultó de la descentralización), pero que asumen la provisión de servicios de salud como parte de su agenda de gobierno, en un contexto en el que los gobiernos nacionales se desentienden de la salud. La concentración de población y su retraso histórico en el reparto de recursos federales, convierten a la provincia de Buenos Aires y, en particular, a los veinticuatro municipios del Gran Buenos Aires, en un caso de análisis acerca de la dinámica derivada de asumir responsabilidades en la provisión de servicios en un contexto de fragilidad financiera.

Como sucede en el resto de la provincia, en el Gran Buenos Aires la descentralización siguió inercialmente "hacia abajo", abriendo el juego a los municipios, delimitando un campo (el de salud) que se fue constituyendo a partir de un doble juego de fuerzas: "desde arriba", a través de procesos clásicos de transferencia de servicios desde el estado provincial; y “desde abajo” por la asunción de responsabilidades sobre centros de salud y, en algunos casos, también sobre los hospitales. Como consecuencia 


\section{Cuadro 1}

Datos básicos de los entrevistados.

\begin{tabular}{|c|c|c|c|c|c|c|}
\hline Nominación & $\begin{array}{c}\text { Nivel } \\
\text { gubernamental }\end{array}$ & Área/Programa & Puesto & $\begin{array}{c}\text { Período permanencia } \\
\text { en el puesto }\end{array}$ & Profesión & ID \\
\hline $\begin{array}{l}\text { Autoridad } \\
\text { sanitaria }\end{array}$ & Municipio & $\begin{array}{l}\text { Secretaría de Salud y } \\
\text { Desarrollo Social }\end{array}$ & Secretario & $2003-2011$ & Médico & A \\
\hline $\begin{array}{c}\text { Alto } \\
\text { funcionario }\end{array}$ & Municipio & Secretaría de Abordajes Integrales & Secretaria & $2010-2011$ & $\begin{array}{l}\text { Licenciatura en } \\
\text { Psicología }\end{array}$ & B \\
\hline $\begin{array}{l}\text { Autoridad } \\
\text { sanitaria }\end{array}$ & Municipio & $\begin{array}{l}\text { Secretaría de Salud y } \\
\text { Desarrollo Social }\end{array}$ & Secretario & $2003-2011$ & Médico & C \\
\hline $\begin{array}{c}\text { Alto } \\
\text { funcionario }\end{array}$ & Municipio & $\begin{array}{l}\text { Secretaría de Salud y } \\
\text { Desarrollo Humano }\end{array}$ & Secretario & $2003-2010$ & Médico & $\mathrm{D}$ \\
\hline $\begin{array}{c}\text { Alto } \\
\text { funcionario }\end{array}$ & Municipio & Secretaría de Salud & Secretario & 2008 a la actualidad & Médico & $\mathrm{E}$ \\
\hline $\begin{array}{c}\text { Alto } \\
\text { funcionario }\end{array}$ & Municipio & Secretaría de Salud & $\begin{array}{l}\text { Secretario y } \\
\text { subsecretario }\end{array}$ & $2003-2007$ & Médico & $\mathrm{F}$ \\
\hline $\begin{array}{c}\text { Alto } \\
\text { funcionario }\end{array}$ & Municipio & Secretaría de Salud & Secretario & 2007 a la actualidad & Médico & G \\
\hline $\begin{array}{c}\text { Alto } \\
\text { funcionario }\end{array}$ & Municipio & $\begin{array}{l}\text { Secretaría de Desarrollo } \\
\text { Social y Salud }\end{array}$ & Secretaria & $2001-2007$ & Médico & $\mathrm{H}$ \\
\hline $\begin{array}{l}\text { Autoridad } \\
\text { sanitaria }\end{array}$ & Municipio & Secretaría de Salud del Municipio & Director & $2001-2007$ & Médico & 1 \\
\hline $\begin{array}{c}\text { Alto } \\
\text { funcionario }\end{array}$ & Municipio & $\begin{array}{c}\text { Secretaría de Desarrollo Social y } \\
\text { Humano }\end{array}$ & Secretario & $2010-2011$ & $\begin{array}{l}\text { Licenciatura en } \\
\text { Psicología }\end{array}$ & $\mathrm{K}$ \\
\hline $\begin{array}{l}\text { Autoridad } \\
\text { sanitaria }\end{array}$ & Municipio & Secretaría de Salud del Municipio & Secretario & $1997-2011$ & Médico & L \\
\hline $\begin{array}{c}\text { Alto } \\
\text { funcionario }\end{array}$ & Municipio & Secretaría de Salud & Subsecretaria & 2008 a la actualidad & Médico & M \\
\hline $\begin{array}{c}\text { Alto } \\
\text { funcionario }\end{array}$ & Municipio & Secretaría de Salud & Secretario & 2008 a la actualidad & Médico & $\mathrm{N}$ \\
\hline $\begin{array}{c}\text { Alto } \\
\text { funcionario }\end{array}$ & Municipio & Secretaría de Salud & Directora & $2002-2005$ & Odontóloga & J \\
\hline $\begin{array}{c}\text { Alto } \\
\text { funcionario }\end{array}$ & Provincia & Maternidad e Infancia/Plan Nacer & $\begin{array}{l}\text { Coordinadora } \\
\text { provincial }\end{array}$ & 2010 a la actualidad & Médica pediatra & \\
\hline $\begin{array}{c}\text { Alto } \\
\text { funcionario }\end{array}$ & Provincia & $\begin{array}{l}\text { Ministerio de Economía de la } \\
\text { Provincia de Buenos Aires }\end{array}$ & $\begin{array}{c}\text { Subsecretario de } \\
\text { Coordinación Fiscal }\end{array}$ & 2003-2007 & Contador & \\
\hline $\begin{array}{c}\text { Alto } \\
\text { funcionario }\end{array}$ & Nación & Programa Remediar & $\begin{array}{l}\text { Coordinadora } \\
\text { nacional }\end{array}$ & 2002 a la actualidad & Contador & \\
\hline $\begin{array}{c}\text { Alto } \\
\text { funcionario }\end{array}$ & Nación & Plan Nacer & $\begin{array}{l}\text { Coordinador } \\
\text { nacional }\end{array}$ & 2007 a la actualidad & Contador & \\
\hline $\begin{array}{c}\text { Alto } \\
\text { funcionario }\end{array}$ & Provincia & Plan Nacer & $\begin{array}{l}\text { Coordinadora } \\
\text { provincial }\end{array}$ & 2010 a la actualidad & Médica pediatra & \\
\hline $\begin{array}{c}\text { Alto } \\
\text { funcionario }\end{array}$ & Nación & Programa Remediar & $\begin{array}{l}\text { Coordinador } \\
\text { Nacional }\end{array}$ & $2007-2009$ & Médico & \\
\hline $\begin{array}{l}\text { Autoridad } \\
\text { sanitaria }\end{array}$ & Provincia & $\begin{array}{l}\text { Subsecretaría de Coordinación } \\
\text { y Atención de la Salud de la } \\
\text { Provincia de Buenos Aires }\end{array}$ & Subsecretario & $2009-2011$ & Médico & \\
\hline $\begin{array}{c}\text { Alto } \\
\text { funcionario }\end{array}$ & Provincia & Programa Remediar & Director & 2008 a la actualidad & Médico & \\
\hline
\end{tabular}


de estos cambios institucionales, al tiempo que se consolidaba un lugar de relativa jerarquía para los municipios en el campo de la provisión de salud en la década previa a la crisis de 2001, el estado provincial se fue especializando sobre lo hospitalario (Tabla 1).

En el contexto de estos procesos, la "salud" fue formando parte de la agenda de gobierno de los jefes comunales (como sucedió con los gobernadores en décadas anteriores) y, en consecuencia, el papel ejercido por los municipios en este campo se fue enhebrando en los procesos de construcción de legitimidad político electoral de los gobiernos locales, a pesar de la fragilidad que también caracterizó a las finanzas municipales.

La trayectoria institucional de esta "historia larga" de conformación del subsector estatal en el Gran Buenos Aires delimitó un esquema de distribución de funciones, que tuvo por responsables principales al Estado provincial (especializado en la provisión de los servicios hospitalarios) y a los municipios, a cargo de otra parte de los hospitales y de los servicios del primer nivel de atención. Este esquema distingue a estos dos niveles de gobierno en la función de provisión de servicios de salud respecto al nacional (casi sin establecimientos a cargo) en los problemas que formarán parte de sus agendas.

Sobre los legados de esa "historia larga", se escribió otra más "reciente" a partir de la crisis social, económica y política de comienzo de siglo que, en sus efectos particulares, produjo un incremento de la demanda sobre el subsector estatal, puso "en jaque" las finanzas en todos los niveles de gobierno e interpeló en su legitimidad a autoridades e instituciones gubernamentales.

La crisis de principios de siglo no parece haber impactado de manera homogénea en el margen de acción de los actores gubernamentales. Determinados por su trayectoria, sus consecuencias económicas y políticas parecen haber reforzado las diferencias pre-existentes entre niveles de gobierno. Mientras el Ministerio nacional (con apenas muy pocos establecimientos a cargo) logró liderar una agenda centrada en la atención primaria de la salud en el escenario intergubernamental, las decisiones provinciales y también municipales estuvieron "amarradas" al imperativo de garantizar la provisión de los servicios en los establecimientos a su cargo.

En el caso particular de los municipios, la agenda en salud estuvo atravesada por problemas estructurales que fueron resolviendo de diferente modo; nos referimos al financiamiento de los servicios frente a una demanda creciente, a la gestión de recursos humanos (que comprende contar los equipos profesionales suficientes en centros de salud y hospitales, bajo las modalidades de contratación disponibles en el empleo público) y a la resolución de las derivaciones hacia niveles de mayor complejidad.

Las respuestas locales a esta agenda dependieron no sólo de la capacidad institucional de cada municipio, sino muy especialmente de sus condiciones financieras, que se correlacionan de manera directa a la situación socioeconómica de su población.

Sin establecimientos a su cargo, la política sanitaria nacional logró liderar una agenda basada en la Atención primaria de la salud e institucionalizada en el Plan Federal de Salud, aprobado en el año 2004, que abordó el problema de la fragmentación del sistema y la desigualdad entre sectores sociales y territorios. En ese contexto general, seleccionamos dos dispositivos de política que tuvieron por preocupación cerrar las brechas en las condiciones bajo las cuales se hace efectivo el derecho a la salud en los establecimientos del subsector estatal: el Programa Remediar y el Plan Nacer.

Tabla 1

Camas en establecimientos estatales y establecimientos sin internación (centros de atención primaria de la salud - CAPS), según dependencia administrativa (nación, provincia y municipios), 24 partidos del Gran Buenos Aires y resto de la provincia, Argentina, 2011.

\begin{tabular}{|c|c|c|c|c|c|c|c|c|}
\hline \multirow[t]{2}{*}{ Dependencia } & \multicolumn{4}{|c|}{ Camas en establecimientos con internación } & \multicolumn{4}{|c|}{ Establecimientos sin internación } \\
\hline & Total & Nación & Provinciales & Municipales & Total & Nación & Provinciales & Municipales \\
\hline Total provincia & 27.660 & 1.756 & 12.350 & 13.554 & 1.993 & 1 & 120 & 1.872 \\
\hline Total Gran Buenos Aires & 9.144 & 451 & 5.581 & 3.112 & 806 & 0 & 34 & 772 \\
\hline Resto de la provincia & 18.516 & 1.305 & 6.769 & 10.442 & 1.187 & 1 & 86 & 1.100 \\
\hline
\end{tabular}

Fuente: Ministerio de Salud de la Provincia de Buenos Aires, Dirección de Información Sistematizada. 
Como parte de la política de medicamentos, el Programa Remediar comprendió la provisión y distribución gratuita de medicamentos esenciales ambulatorios en los centros de atención primaria de la salud (CAPS). Destinado a la población con cobertura de salud pública exclusiva, las rutinas y procedimientos no parecen haber impedido la entrega del medicamento a toda la población que acudía a ser atendida en los CAPS. A través de la entrega de los denominados "botiquines", el Programa buscó fortalecer la capacidad resolutiva del primer nivel y capacitar a los equipos en la estrategia de atención primaria de la salud. La distribución de los "botiquines" era centralizada y directa a los CAPS. La gratuidad en la consulta era uno de los requisitos que el programa establecía a los establecimientos.

El Plan Nacer fue un dispositivo de la política nacional, orientado a formar seguros públicos provinciales destinados a garantizar un conjunto de prestaciones a la población sin cobertura explícita en salud. En sus orígenes, buscó revertir el aumento de las tasas de morbi-mortalidad materna e infantil y proveer garantías explícitas para la población vulnerable, dirigiéndose a embarazadas y niños/as menores de 5 años. Estaba basado en un sistema de incentivos monetarios asociados a resultados; distintos instrumentos vinculaban las transferencias monetarias a haber alcanzado resultados en cobertura poblacional, prestaciones a cubrir y metas sanitarias denominadas "trazadoras". En el marco de estos compromisos, cada efector recibía recursos financieros que podían destinar a la construcción y mejoras edilicias, a la compra y mantenimiento de equipamiento, a la contratación y capacitación de recursos humanos o a la compra de insumos no provistos por otros planes.

Estos dispositivos expresaron intentos por producir cambios en los problemas que estructuraban la atención; cada uno de ellos, representaba una determinada "hoja de ruta" del cambio que, más allá de las diferencias, compartían un atributo en común: el afán por recuperar la capacidad regulatoria sobre el subsector estatal sin modificar el esquema de distribución de funciones resultante de la descentralización.

La apuesta a introducir cambios orientados a restaurar condiciones más igualitarias en el acceso a derechos enfrentó singulares desafíos en el escenario fragmentado del Gran Buenos Aires. Las dimensiones que propone la literatura para analizar los movimientos hacia la universalidad en salud permiten reconstruir los retos que enfrentó la política sanitaria: el acceso (considerando las condiciones que lo facilitan y la gratuidad en la atención); la cobertura (en términos del alcance poblacional); y los beneficios (prestaciones brindadas en cantidad y calidad) 15 .

En torno a los retos de la política sanitaria, se produjeron nociones e ideas en el nivel de la implementación municipal que dialogaban y entraban en tensión con las argumentaciones técnicas en las que se fundaban los dispositivos de políticas.

\section{Nociones e ideas en torno a la implementación}

En sus diagnósticos, objetivos e instrumentos, así como también en la definición de la población merecedora de los beneficios, estos dispositivos condensaron distintas nociones e ideas acerca de los cambios en la práctica de la atención en el nivel municipal.

En la singularidad del contexto institucional que caracteriza al caso bajo estudio, y conforme las orientaciones que expresan estos dispositivos de políticas, interesa ahora indagar en cómo se conciben estos intentos de cambio en los modelos de atención. Para responder a esta pregunta es necesario trascender los discursos técnicos de los programas, y detectar las convergencias, divergencias y tensiones que se construyen en torno a las ideas que expresan estos dispositivos.

Nuestra mirada recupera las argumentaciones de los promotores de los dispositivos, y muy especialmente de los funcionarios municipales (casi en su totalidad secretarios de salud), "mediadores" en palabras de Muller 7 entre las iniciativas supra locales y la responsabilidad sobre los servicios.

La apuesta de introducir cambios orientados a restaurar condiciones más igualitarias en el ejercicio de derechos enfrenta singulares desafíos en una región que ha sido escenario de una profunda fragmentación. En ese contexto, las políticas fueron perfilando tres retos: "facilitar el acceso a los servicios", "asegurar la cobertura de servicios de salud" y "garantizar beneficios de manera explícita". (a) La facilitación en el acceso a los servicios: la erradicación de las barreras económicas (Cuadro 2).

La apuesta de la política pública nacional sobre el primer nivel de atención encontró en el Gran Buenos Aires una trama vasta y heterogénea de centros de salud y puestos sanitarios que presentaba distintas formas de mercantilización en el acceso a los servicios; el cobro del "bono contribución" era 


\section{Cuadro 2}

Reto (a) -facilitación en el acceso a los servicios.

\begin{tabular}{|c|c|}
\hline Dimensiones & Perspectiva de los actores \\
\hline \multirow{3}{*}{$\begin{array}{l}\text { Lecturas acerca } \\
\text { del cobro } \\
\text { del "bono } \\
\text { contribución" }\end{array}$} & $\begin{array}{l}\text { "Hasta junio del } 2002 \text { no había un centro de salud que no cobrara: todos tenían un bono cooperadora. Todos los municipios hacían } \\
\text { un negocio con la salud. Y ahí fue claro, para recibir los medicamentos gratis no se cobra" (EV Alto funcionario nacional). }\end{array}$ \\
\hline & $\begin{array}{c}\text { "La pelea contra los bonos es ancestral (...) ‘No.., si nosotros no cobramos, la atención es gratuita, el bono es totalmente voluntario, } \\
\text { paga el que quiere' (...) Lo que pasa es que la conciencia de gratuidad existe, lo que no existe es la conciencia que el bono es una } \\
\text { barrera" (EV Alto funcionario provincial). }\end{array}$ \\
\hline & $\begin{array}{l}\text { "Este es un municipio donde hubo cooperadora, la cooperadora es casi clásica en los centros de salud (...) Sobre todo cuando el } \\
\text { hospital estaba en [NOMBRE DEL PARTIDO], la población de la zona oeste venía al hospital: un viaje para sacar un turno, un segundo } \\
\text { viaje para ir a ver al médico, un tercer viaje para hacerse los estudios que le pedía, un cuarto para retirarlos, un quinto para ir a } \\
\text { verlo al médico de nuevo. Es decir, prefiero pagar cinco pesos en el centro de salud y me vea un médico de ahí, por lo cual había } \\
\text { profesionales que estaban por la cooperadora que cobraban esa cifra" (EV Alto funcionario municipal I). }\end{array}$ \\
\hline \multirow[t]{3}{*}{$\begin{array}{l}\text { Gratuidad } \\
\text { como } \\
\text { requisito de } \\
\text { los programas } \\
\text { nacionales }\end{array}$} & $\begin{array}{c}\text { “Cerraban el centro de salud donde ellos comprobaban que se había cobrado alguna prestación. Entonces [el Programa Remediar] } \\
\text { acelera, porque en los centros de salud municipales había cooperadoras y otros eran de gestión mixta, la sociedad de fomento, } \\
\text { algunos tenían podología, masajista y hacían un pesito con eso... Para ellos [Programa Remediar] no distinguían esto... ¿cobran } \\
\text { en el centro? Y sí, cobran. (...) Entonces al empezar a ser muy rígidos y cerrar la provisión de medicamentos..." (EV Alto funcionario } \\
\text { municipal D). }\end{array}$ \\
\hline & $\begin{array}{l}\text { "Se cobraban bonos voluntarios hasta que apareció Remediar. El Remediar prohíbe el cobro de cualquier tipo de bono. Y ahí se cortó" } \\
\text { (EV Alto funcionario municipal J). }\end{array}$ \\
\hline & $\begin{array}{c}\text { "A nosotros el Remediar, entre otras cosas, nos ayudó para erradicar los bonos de consulta de la totalidad de los centros. Era } \\
\text { un combate que veníamos dando porque era complejo. No quedó una alcancía, no quedó nada. Fue letal el Remediar" (EV Alto } \\
\text { funcionario municipal K). }\end{array}$ \\
\hline $\begin{array}{l}\text { Alineamiento } \\
\text { de rutinas en } \\
\text { los centros } \\
\text { de atención } \\
\text { primaria de la } \\
\text { salud }\end{array}$ & $\begin{array}{c}\text { "A nosotros el Remediar entre otras cosas nos ayudó para erradicar los bonos de consulta de la totalidad de los centros. Era un } \\
\text { combate que veníamos dando porque era complejo. No quedó una alcancía, no quedó nada. Fue letal el Remediar" (EV Alto } \\
\text { funcionario municipal K). }\end{array}$ \\
\hline \multirow[t]{2}{*}{$\begin{array}{l}\text { Limitaciones de } \\
\text { la gratuidad }\end{array}$} & $\begin{array}{l}\text { "Las municipalidades se deben a sus vecinos, traducido: para quien viva en [NOMBRE DEL MUNICIPIO] es el verdadero efector } \\
\text { gratuito que yo le tengo que dar el servicio. Yo no voy a poner luz en San Miguel, ni voy a asfaltar en Pilar (...) Consecuentemente, } \\
\text { por qué debo dar salud a otros municipios... ¿Simplemente por una cuestión de solidaridad? Pero en la práctica mi obligación legal } \\
\text { está ligada directamente a mis vecinos. Conclusión, ningún paciente de [NOMBRE DEL MUNICIPIO] pasa por la cooperadora y todo } \\
\qquad \text { paciente que venga de otro distrito pasa por la cooperadora" (EV Alto funcionario municipal L). }\end{array}$ \\
\hline & $\begin{array}{c}\text { "Hay algunos hospitales municipales donde esto es clásico, de los municipales que tenemos en la zona hay un municipio en el que no } \\
\text { tenemos problemas que es vecino a la General Paz y otro que está vecino a él en el que tradicionalmente no se atiende a gente que } \\
\text { no es del municipio. Sí en la guardia y sí se interna... o sea, una actitud muy generosa en eso, pero en lo programado no" (EV Alto } \\
\text { funcionario municipal I). }\end{array}$ \\
\hline
\end{tabular}

Fuente: elaboración propia.

la modalidad más extendida. Mientras en algunos casos estaba promovido desde los gobiernos locales, en el resto se originaba y era administrado por los mismos centros con distinto nivel de aceptación por parte de las autoridades municipales.

En este contexto, la formalización de la gratuidad como requisito para mantenerse como instancia de implementación, junto a las auditorias del Programa Remediar, fueron los instrumentos a través de los cuales se libró la batalla por la remoción del "bono" como barrera económica en el acceso; el Plan Nacer parece haber capitalizado esos resultados y sostenido el requisito.

El diagnóstico de situación acerca del fenómeno de mercantilización en el acceso variaba según la distancia de los actores respecto de la responsabilidad sobre la provisión de los servicios. Los testimo- 
nios muestran contrapuntos entre niveles de gobierno, argumentos que van ganando en complejidad, a medida que se acercan a la gestión de los servicios, con diferencias también en la relevancia que le confieren al bono como "barrera" en el acceso.

Aun partiendo de distintas justificaciones (financiamiento de especialistas, a cargo de entidades intermedias, pago a profesionales que trabajan en contraturno, entre otras), los testimonios de los secretarios de salud municipales entrevistados coinciden en valorar la importancia que tuvo el Programa Remediar para corregir procesos de mercantilización. Mientras algunos lo visualizaron como una vía para intervenir positivamente en la remoción de las barreras en el acceso, otros parecen haber estado obligados a modificar políticas locales basadas en el arancelamiento.

La eficacia del Programa Remediar parece haberse librado en dos planos. El primero remite a la preexistencia de un marco de ideas propicio y compartido sobre la "gratuidad en la atención de la salud", presente en los actores del subsector estatal, incluso a pesar de las transformaciones de la década de los noventa. El segundo plano es político, y se expresa en el significado que tuvo para los gobiernos locales la posibilidad de alinear las rutinas de los CAPS (de origen mixto y muchas veces bajo control de las organizaciones sociales) en la dinámica de la política estatal.

La idea de que el cobro de un bono para la atención es una barrera, "viaja" en las "hojas de ruta" de los dispositivos (programmes) y encuentra un marco de ideas propicio acerca de la gratuidad en los secretarios de salud municipales. Pareciera que forma parte del policy paradigm, compartido por los actores en los distintos niveles de gobierno durante este periodo.

(b) El aseguramiento de la cobertura: ¿quiénes son los destinatarios de los servicios estatales? (Cuadro 3).

\section{Cuadro 3}

Reto (b) -aseguramiento de la cobertura.

\begin{tabular}{|c|c|}
\hline Dimensiones & Perspectiva de los actores \\
\hline \multirow[t]{2}{*}{$\begin{array}{l}\text { Limitaciones } \\
\text { del modelo de } \\
\text { aseguramiento }\end{array}$} & $\begin{array}{l}\text { "El Plan Nacer es una 'pata' de financiamiento para nosotros, lo que importa es el Programa Materno Infantil. Entonces a mí no me } \\
\text { importa que tenga seguridad social, ya que las prestaciones, las cosas que uno hace es lo mismo (...) Yo creo que es muy bueno que } \\
\text { se financie, porque además entonces tenés toda la población con financiamiento. (...) Para mí, iguala, o sea a mí no me importa si } \\
\text { tiene o no tiene cobertura en la seguridad social" (EV Alto funcionario municipal E). }\end{array}$ \\
\hline & $\begin{array}{l}\text { "En general la población que se atendía en los CAPS no tenía otra cobertura que no sea la estatal, así que eso... a nosotros se nos } \\
\text { jugaba más en las restricciones etarias, no tanto en el tema de la cobertura" (EV Alto funcionario municipal K). }\end{array}$ \\
\hline $\begin{array}{l}\text { Labilidad de las } \\
\text { fronteras entre } \\
\text { subsectores }\end{array}$ & $\begin{array}{l}\text { "Hay una falacia en sostener que el primer nivel de atención o el sector público es para quienes no tienen cobertura. En el } \\
\text { programa de diabetes, el cuarenta por ciento de los diabéticos eran Programa Materno Infantil-dependientes, y venían a buscar el } \\
\text { medicamento al centro de salud [municipal], porque es el efector más cercano. Aunque el Programa Materno Infantil I le cubría } \\
\text { como prestador el 100\% de la prestación (....)" (EV Alto funcionario municipal N) }\end{array}$ \\
\hline \multirow[t]{4}{*}{$\begin{array}{l}\text { La nominalización } \\
\text { como instrumento }\end{array}$} & $\begin{array}{c}\text { "La nominalización de la población nos sirve absolutamente, porque se registra en nuestro sistema. Lo que tenemos que } \\
\text { solucionar es el tema de esta cosa de hacer compatibles las bases y todo eso, que no sé si será posible en algún momento" (EV Alto } \\
\text { funcionario municipal E) }\end{array}$ \\
\hline & $\begin{array}{c}\text { "Es un problema porque cada programa tiene su modalidad de registro, sus condiciones de carga, y ahí hay un menú de } \\
\text { posibilidades, desde que el municipio solamente cargue y no reciba nunca más información de eso que generó como dato, o } \\
\text { que sí, o que lo tengas que cargar y después tengas como un resumen, una síntesis de lo que vos cargaste, o sea, eso si" (EV Alto } \\
\text { funcionario municipal B) }\end{array}$ \\
\hline & $\begin{array}{c}\text { "Con el Plan Nacer lo que no tenemos es la información. Nosotros lo que tenemos es un millón de listados, informatizados y qué sé } \\
\qquad y o, \text { que se los mandamos vía web..." (EV Alto funcionario municipal K) }\end{array}$ \\
\hline & $\begin{array}{l}\text { "Es complejo el tema de la nominalización. Nosotros a lo que tratamos de ir es a que cada centro de salud tenga lo más claro } \\
\text { posible su área programática y también la población que vive en esa área programática. Hay varios mecanismos, sobre todo } \\
\text { materno infantiles, un poco menos en adultos, de seguimiento domiciliario de casos, y eso es por área programática; digamos, ahí } \\
\text { la nominalización es súper concreta" (EV Alto funcionario municipal K) }\end{array}$ \\
\hline
\end{tabular}

Fuente: elaboración propia. 
La persistencia de un núcleo de población sin otro acceso a servicios de salud que el provisto por el subsector estatal indujo a desplegar dispositivos orientados a ampliar la cobertura poblacional. En los dispositivos analizados, esta preocupación se expresaba en la definición de su población objetivo (que no tuviera obra social o seguro privado), a través del empadronamiento de la población y de la nominalización de la atención. Recuperar la mirada de los actores permite dar cuenta de otros pliegues y abrir nuevas preguntas.

Funcionarios de los tres niveles coinciden en la pertinencia de restringir la asignación de las transferencias financieras (hacia los municipios) a la población que carece de otra cobertura de salud; se trata de un instrumento utilizado por el Plan Nacer y, más débilmente, el Programa Remediar.

Aun partiendo de estas coincidencias, esta condición era vista como necesaria (y legítima) para obtener un financiamiento adicional de parte de la nación y la provincia, pero no como un atributo desde el cual redefinir el alcance de los servicios.

La lógica de aseguramiento prevista en el Plan Nacer encuentra en la implementación en el Gran Buenos Aires una muy desigual distribución en el territorio de los prestadores de la seguridad social; así, en los municipios del segundo cordón del Gran Buenos Aires priman los establecimientos estatales sobre los prestadores de la seguridad social.

El desafío de alcanzar la cobertura de la totalidad de la población parece ser una idea compartida entre los distintos niveles gubernamentales (lo que podría inscribirse en el policy paradigm). La definición de la población sin otra cobertura que la estatal es un criterio aceptado para validar las transferencias hacia los municipios; sin embargo, no alcanza a delimitar a la población destinataria de los servicios estatales, especialmente los del primer nivel de atención.

Las voces de los secretarios de salud municipales introducen otra perspectiva que anida en la noción de "derecho" de todos los "vecinos" del municipio a la prestación pública de salud (en el plano de lo que se denomina frame, realimentado por los policy sentiments), que contrasta con la lógica de los modelos de aseguramiento que organiza la "hoja de ruta" del Plan Nacer (programmes), poniendo en discusión incluso parte de sus instrumentos.

En un plano estrictamente político, los gobiernos locales parecen sostener como destinatario a un sujeto que "funde" su demanda de salud con otras demandas sociales y políticas, noción que interpela la idea de "nominalidad" recortada exclusivamente a la población sin cobertura.

(c) Garantía de beneficios explícitos: disputas en torno a los instrumentos (Cuadro 4).

Los dispositivos de políticas recorren muy diversas instituciones estatales, situación que advierte acerca de la importancia y, a la vez, la complejidad que implica garantizar un conjunto de beneficios en calidad y cantidad que sea homogéneo para toda la población en el subsector estatal, independientemente del lugar de residencia.

Se trata de un desafío abordado (con intensidad y alcance variable) por ambos programas, a través de un conjunto de incentivos que buscaron regular el margen de discrecionalidad de los actores en el nivel subnacional y definir beneficios homogéneos, dotando de protagonismo a los establecimientos, especialmente a aquellos pertenecientes al primer nivel de atención.

Se trataba de uno de los retos más complejos ya que -en el Gran Buenos Aires- enfrentó muy diversas situaciones que encontraron dificultades para ser "atrapadas" a través de las "hojas de ruta" de los dispositivos: el Programa Remediar que apuntaba al fortalecimiento de la oferta a través de la provisión de medicamentos esenciales, mientras que el Plan Nacer buscaba garantizar beneficios homogéneos, separando la función de compra de la función de provisión de servicios.

Una primera cuestión a considerar es que la implementación supuso atravesar el nivel municipal de toma de decisiones, interpelado por una agenda de problemas estructurales que involucraba cuestiones relativas al financiamiento (por lo cual el ingreso a estos planes y programas nunca fue objeto de discusión), al sostenimiento de los equipos profesionales en los establecimientos (lo que denominamos genéricamente gestión de recursos humanos) y a la coordinación para la resolución de derivaciones. La instrumentación de la "responsabilidad nominada" y de los distintos sistemas de pago se vieron atravesados por las cuestiones comprendidas en esta agenda.

La visión de los promotores de los dispositivos está alineada con la lógica técnica del diseño, mientras que en el nivel local se advierten otros clivajes derivados del desafío de cumplir con las condiciones que les imponen los programas, al tiempo que buscan resolver -a través de sus recursos- los problemas estructurales que enfrentan como responsables de la provisión de los servicios. 


\section{Cuadro 4}

Reto (c) -garantía de beneficios explícitos en salud.

\begin{tabular}{|c|c|}
\hline Dimensiones & Perspectiva de los actores \\
\hline \multirow[t]{2}{*}{$\begin{array}{l}\text { Brechas entre } \\
\text { el diseño y la } \\
\text { implementación } \\
\text { de los programas }\end{array}$} & $\begin{array}{c}\text { "...en un contexto hiper descentralizado todas las provincias están trabajando con el mismo paquete prestacional, con el mismo } \\
\text { indicador de desempeño, con el mismo esquema de auditoría, con la misma estrategia de comunicación, con la misma población } \\
\text { objetivo... Es un gran efecto ordenador de alinear esfuerzos, de concentrar esfuerzos para obtener un resultado superador" (EV } \\
\text { Alto funcionario nacional). }\end{array}$ \\
\hline & $\begin{array}{c}\text { "Hacer salud es como hacer matemática compleja, y los programas son las tablas de multiplicar. Está bien, si no conocés las } \\
\text { tablas no podés hacer matemática compleja, pero no te creas que sabiendo las tablas estás haciendo salud" (EV Alto funcionario } \\
\text { municipal N). }\end{array}$ \\
\hline \multirow{2}{*}{$\begin{array}{l}\text { Relación } \\
\text { causal entre } \\
\text { "producción"y } \\
\text { "resultados" }\end{array}$} & $\begin{array}{l}\text { “Los equipos están más preocupados por lo que facturan que por las trazadoras que cumplen o no (EV Alto funcionario } \\
\text { municipal N). }\end{array}$ \\
\hline & $\begin{array}{c}\text { "Hoy la trazadora sigue siendo una situación administrativa, o sea no está en la discusión con los equipos, ni siquiera en la } \\
\text { discusión de los programas. Te digo más: "Estamos abajo en tal trazadora, fíjense cómo mejoran el registro", casi, no están en "che, } \\
\text { se nos están escapando las embarazadas..." (EV Alto funcionario municipal K). }\end{array}$ \\
\hline \multirow{2}{*}{$\begin{array}{l}\text { Acerca de los } \\
\text { instrumentos de } \\
\text { pago }\end{array}$} & $\begin{array}{c}\text { "El sistema de salud, y el sistema de salud público, es impermeable a la recaudación... hay que trabajarla muchísimo" (EV Alto } \\
\text { funcionario municipal M). }\end{array}$ \\
\hline & $\begin{array}{c}\text { "[El Plan Nacer] Tiene una concepción neoliberal, ¿por qué? Porque hay que facturar, ahí cambió todo. Entonces el profesional cree } \\
\text { que como se está hablando de facturación, le van a pagar a él si entra la plata... Entonces cuesta mucho porque la gente empieza } \\
\text { a hacer las planillas, la facturación, pero después esto tarda como seis meses... entonces no ven la plata..." (EV Alto funcionario } \\
\text { municipal G). }\end{array}$ \\
\hline $\begin{array}{l}\text { Los incentivos } \\
\text { financieros } \\
\text { y la recursos } \\
\text { humanos }\end{array}$ & $\begin{array}{l}\text { "Entonces terminás usándolo como incentivo y terminan siendo sobresueldos por el mismo número de prestación que debería } \\
\text { hacer... (...) Los programas que hablan de incentivos al recurso humano, están fragmentado por programa, no te permite tener una } \\
\text { política de recurso humano, no te permite tener una política clara de incentivo 'para qué..." (EV Alto funcionario municipal N). }\end{array}$ \\
\hline
\end{tabular}

Fuente: elaboración propia.

La "responsabilidad nominada" está presente en el Plan Nacer, buscando asegurar el cumplimiento de determinadas metas en la situación de salud de la población atendida.

A diferencia de lo que pudimos advertir con relación a las barreras de acceso y la ampliación de la cobertura, la adopción de instrumentos orientados hacia la separación de funciones entra en tensión con un marco de ideas (compartida entre los secretarios municipales) que estaba fundado en el objetivo de fortalecer la oferta estatal.

La lectura que hacen los funcionarios municipales cuestiona los argumentos técnicos en los que se sostienen estos esquemas de incentivos. Los relatos aluden a que la motivación por la facturación se sobrepone a la necesidad de responsabilización de los equipos de salud en el cumplimiento de las metas. Desde esta perspectiva, la relación causal entre "producción" (expresada en la facturación) y "resultados sanitarios" parece haber quedado relegada, al menos en los discursos de estos actores, a una "trastienda" meramente administrativa.

Los instrumentos de pago ("pago capitado” y "por prestación”) se resisten desde la óptica de los secretarios de salud, ya que tensionan con el modelo de financiamiento de la oferta que encarna la organización de los servicios municipales. Las posiciones más escépticas se fundan en diferencias con el paradigma de políticas, en la falta de confianza de los profesionales o bien en el potencial distorsivo que, sobre las prácticas, tiene este incentivo.

La jerarquía que tienen estos instrumentos de pago en la "hoja de ruta" de los dispositivos tiende a diluirse en el contexto de la implementación, atravesado por una agenda vinculada a la gestión de los recursos humanos y encuentra al municipio como actor responsable de la asignación para garantizar la provisión de los servicios. 
En los relatos de los secretarios municipales, las dificultades para completar equipos frente a una demanda creciente es uno de los problemas que interpela al sistema de pago de los dispositivos organizados bajo el modelo de aseguramiento. Alejado del horizonte supuesto en los argumentos técnicos que avalan los programas, el completamiento de salarios para retener profesionales representa otra de las formas de adaptación en que derivan estos instrumentos.

El análisis del recorrido de los dispositivos, en relación a la apuesta por garantizar beneficios homogéneos en calidad y cantidad para el conjunto de la población, muestra cómo la teoría causal en la que se basan los dispositivos, se pone en cuestión en la implementación, no sólo a partir del diálogo (y la tensión) con otras ideas, sino en los procesos de toma de decisiones expresados en la agenda de problemas que comparten los secretarios de salud municipales.

\section{Conclusión}

Como puede advertirse del recorrido realizado en este trabajo, los programas de salud salen de las "tranquilas aguas" de su diseño y transitan el camino de la instrumentación en el registro de los actores; en ese recorrido, cada nivel gubernamental se distingue por las ideas desde las cuales se construyen socialmente los cambios, los problemas que configuran su agenda y por el contexto en que se toman las decisiones.

El análisis precedente muestra que en la implementación se producen relaciones virtuosas y también tensiones que resultan del modo en el que se retroalimentan las ideas presentes en las "hojas de ruta" de los dispositivos (programmes) con otras -menos evidentes y con mayor consolidación- en el plano de las políticas (policy paradigms), además de las tensiones que se producen cuando éstas colisionan con percepciones compartidas de la sociedad (public sentiments), que se expresan a su vez en símbolos y conceptos que los actores municipales emplean para legitimar su práctica en la implementación (frames).

Esta dinámica se advierte en el modo como sedimentan las ideas en torno a la gratuidad en el acceso, aunque vuelven a legitimarse formas de mercantilización cuando esta idea colisiona con otras, derivadas de una dinámica extra sectorial, que anida en la lógica de acumulación política sobre su población de referencia, el "vecino" y/o "contribuyente".

La ampliación de la cobertura es otro núcleo de convergencia de los actores en el plano del policy paradigm; sin embargo, esta idea refracta especialmente en los dispositivos organizados en la idea de aseguramiento con otras nociones referidas al "derecho" de todos los vecinos del municipio a la prestación pública de salud (en el plano de lo que se denomina frame) de un destinatario que "funde" su demanda de servicios de salud con otras, sociales y políticas.

A diferencia de lo que sucede en relación al acceso y la ampliación de la cobertura, la separación de funciones como supuesto organizador de las políticas (policy paradigms), orientadas a garantizar un conjunto de beneficios de manera homogénea, colide con la idea compartida por los secretarios municipales en relación al fortalecimiento de la oferta estatal.

En relación a este desafío, se pone en evidencia que la implementación es una construcción social compleja que resulta tanto de lo que sucede en el plano de las ideas, como de los procesos de toma de decisiones que están determinados por la matriz institucional resultante de la descentralización que dejó al nivel municipal "amarrado" a la responsabilidad sobre la provisión de los servicios. En torno a la agenda de problemas estructurales que comparten los secretarios de salud municipales, se alinean, tensionan y re-significan las propuestas de cambio originadas en niveles supra locales. 


\section{Agradecimientos}

Agradezco al Fondo Nacional de Investigación Científica y Tecnológica y al Instituto del Conurbano de la Universidad Nacional de General Sarmiento que hicieron posible el proyecto en cuyo contexto se elaboró este trabajo (PICT 0693/2014).

\section{Referencias}

1. Obinger H, Leibfried S, Castles FG, editors. Federalism and the Welfare state: New World and European experiences. Cambridge: Cambridge University Press; 2005.

2. Arretche M. Federalism, Social policy, and reductions of territorial inequality in contemporary Brazil. In: Schneider BR, editor. New order and progress. Development and democracy in Brazil. Oxford: Oxford University Press; 2016. p. 162-84.

3. Pierson P, Skocpol T. El institucionalismo histórico en la ciencia política contemporánea. Revista Uruguaya de Ciencia Política 2008; 17:7-38.

4. Banting $\mathrm{K}$, Corbett $\mathrm{S}$, editors. Health policy and federalism. A comparative perspective. Kingston: Institute of Intergovernmental Relations, Queen's University; 2002.

5. Beland D. Ideas and institutions in social policy research. Soc Policy Adm 2016; 50:734-50.

6. Sabatier P, Weible C. El marco de las coaliciones promotoras. Innovaciones y clarificaciones. In: Sabatier P, editor. Teoría del proceso de políticas públicas, proyecto de modernización del estado. Buenos Aires: Jefatura de Gabinete de Ministros, Presidencia de la Nación; 2010. p. 203-38.

7. Muller P. Las políticas públicas. Estudios de caso n. 3. 3a Ed. Bogotá: Universidad Externado de Colombia; 2010.

8. Kingdon J. Agendas, alternatives and public policies. 2nd Ed. New York: Pearson; 1995. (Longman Classics in Political Science).

9. Laurell AC, Herrera Ronquillo J. La segunda reforma de salud: aseguramiento y compraventa de servicios. Salud Colectiva 2010; 6:137-48.

10. Cotlear D, Gómez-Dantés O, Knaul F, Atun R, Barreto I, Cetrángolo O, et al. Overcoming social segregation in health care in Latin America. Lancet 2015 ; 385:1248-59.

11. Martínez Franzoni J. ¿Presión o legitimación? Poder y alternativas en el diseño y adopción de la reforma de salud en Costa Rica, 1988-1998. Hist Ciênc Saúde-Manguinhos 2006; 3:591622.

12. Santos A M. Giovanella L. Governança regional: estratégias e disputas para gestão em saúde Rev Saúde Pública 2014; 48:622-31.

13. Belmartino $\mathrm{S}$. La atención médica argentina en el siglo XX. Instituciones y procesos. Buenos Aires: Siglo XXI Editores; 2005.

14. Cetrángolo O. Financiamiento fragmentado, cobertura desigual y falta de equidad en el sistema de salud argentino. Revista de Economía Política de Buenos Aires 2014; 13:145-83.

15. Chiara M, Crojethovic M, Ariovich A. El universalismo en salud en Argentina entre 2003 y 2015: balances y desafíos desde una aproximación macro institucional. Salud Colectiva 2017; 13:663-76. 


\section{Abstract}

In the context of a federal and highly fragmented institutional framework like that Argentina, the article analyzes the concepts and ideas on which government actors organize their health policy instruments at three different levels of government. Based on this focus, the article investigates the convergences, divergences, and tensions permeating the exercising of the right to health. The analysis is organized in three dimensions of universal care that became challenges for the national policy during the period in question: ease of access to services, insured coverage, and a guaranteed set of explicit benefits for the entire population. Concerning these challenges, the actors deconstruct and reconstruct the meaning of the policies for universal health care, based on the issues on their agendas, the ideas existing prior to the programs (and based on which the changes are conceived), and the political logic by which their decisions are made. This perspective seeks to transcend the programs' underlying technical ideas in order to capture the political dimension of their implementation, seen as a complex social construction, which also faces structural problems that are part of the agenda at each level of government in relation to health services provision.

Health Policy; Universal Access to Health Care Services; Federalism

\section{Resumo}

No contexto de um órgão institucional federal e fortemente fragmentado como a Argentina, este artigo analisa as noções e ideias sobre as quais os atores governamentais organizam ao redor seus instrumentos de política sanitária em três níveis de governo diferenciados. A partir desta abordagem, foi feita uma pesquisa sobre as convergências, divergências e tensões que atravessam as tentativas de mudanças, orientadas ao restabelecimento de condições mais igualitárias no exercício do direito à saúde. A análise foi organizada em três dimensões de universalidade que foram convertidas em desafios da política nacional durante o periodo analisado: facilitação no acesso aos serviços, garantia de cobertura, além de um conjunto de benefícios explícitos para toda a população. Em torno desses desafios, os atores desconstroem e reconstroem o significado das políticas orientadas pela universalidade em saúde, segundo os problemas que fazem parte da agenda deles, as ideias prévias com a chegada dos programas (e a partir das quais concebem as mudanças) e a lógica política através da qual viabilizam as decisões deles. Esta perspectiva procura transcender as noções técnicas que justificam os programas, a fim de obter a dimensão política da implementação, entendida como uma construção social complexa, que faz frente também a problemas estruturais que compõem a agenda em cada nivel de governo, em relação a provisão de serviços de saúde.

Política de Saúde; Acesso Universal aos Serviços de Saúde; Federalismo
Recibido el 04/Ene/2018

Versión final presentada el 25/May/2018

Aprobado el 12/Jul/2018 